\title{
Interstellar Turbulence and Star Formation
}

\author{
Alexei G. Kritsuk, ${ }^{1}$ Sergey D. Ustyugov, ${ }^{2}$ and Michael L. Norman ${ }^{1}$ \\ ${ }^{1}$ UC San Diego, 9500 Gilman Drive MC 0424, La Jolla CA 92093-0424, USA \\ email: akritsuk@ucsd.edu, mlnorman@ucsd.edu \\ ${ }^{2}$ Keldysh Institute of Applied Mathematics, Miusskaya Sq. 4, 125047, Moscow, Russia \\ email: ustyugs@keldysh.ru
}

\begin{abstract}
We provide a brief overview of recent advances and outstanding issues in simulations of interstellar turbulence, including isothermal models for interior structure of molecular clouds and larger-scale multiphase models designed to simulate the formation of molecular clouds. We show how self-organization in highly compressible magnetized turbulence in the multiphase ISM can be exploited in simple numerical models to generate realistic initial conditions for star formation.
\end{abstract}

Keywords. ISM: structure, ISM: clouds, ISM: magnetic fields, turbulence, methods: numerical

\section{Introduction}

Since most of the ISM is characterized by very large Reynolds numbers, turbulent motions control the structure of nearly all temperature and density regimes in the interstellar gas (Elmegreen \& Scalo 2004). Because of that, turbulence is often viewed as an organizing agent forming and shaping hierarchical cloudy structures in the diffuse ISM and ultimately in star-forming molecular clouds (e.g., Vázquez-Semadeni \& Passot 1999). Nonlinear advection dominating the dynamics of such highly compressible magnetized multi-scale self-gravitating flows makes computer simulations practically the only tool to study fundamental aspects of interstellar turbulence, even though effective Reynolds numbers in numerical models are always limited by the available computational resource (e.g., Kritsuk et al. 2006).

Over the last five years three-dimensional numerical simulations fostered the development of theoretical concepts concerning the interstellar medium undergoing nonlinear self-interaction and self-organization in galactic disks. One can conventionally divide these models designed to tackle various aspects of interstellar turbulence into three different classes depending on the range of resolved scales and physics included: (i) mesoscale models that cover evolution of multiphase ISM in volumes with linear size of a few-to-ten $\mathrm{kpc}$ and resolve the flow structure down to a fraction of $1 \mathrm{pc}$ (e.g., galactic fountain models developed by de Avillez \& Breitschwerdt 2005-07); (ii) sub-mesoscale models resolving the scale-height of the diffuse Hi $(\sim 100 \mathrm{pc})$ and usually limited to only warm-to-cold neutral phases (WNM and CNM) (e.g., Kissmann et al. 2008; Gazol et al. 2009; Gazol \& Kim 2010; Seifried et al. 2010); and (iii) microscale models for molecular cloud (MC) turbulence that assume an isothermal equation of state and deal with $<10$ pc-sized subvolumes within MCs. Global galactic disk models (e.g., Tasker \& Bryan 2006-08, Wada 2008 and references therein) which represent the future of direct ISM turbulence modeling, are currently resolving scales down to $\sim 10 \mathrm{pc}$, i.e. insufficient to properly follow the thermal structure of self-gravitating multiphase ISM.

Mesoscale models of supernova-powered ( $\mathrm{SNe}$ ) galactic fountain have demonstrated the important role of dynamic pressure in the ISM that keeps large fractions of the gas mass out of thermal equilibrium and elevates gas pressures of GMCs to the observed levels 
even without direct action of self-gravity (Korpi et al. 1999; Mac Low et al. 2005; de Avilles \& Breitschwerdt 2005-07; Joung et al. 2006-09). They also show that the effective integral scale of the SN-driven turbulence $(\sim 75 \mathrm{pc})$ is about half the scale height of the HI gas in the inner Galaxy [100 - 150 pc (Malhotra 1995)] and outline a general picture of probability distributions for the mass density, magnetic field strength, and thermal pressure in the turbulent ISM in disk-like galaxies.

Supersonic isothermal turbulence simulations in periodic boxes representative of the microscale models provided many important insights into the physics of interstellar turbulence and helped to guide the interpretation of observations. These numerical experiments highlighted the importance of nonlinear advection as a major feature of compressible turbulence (Pouquet et al. 1991). At high Mach numbers, turbulent flows are dominated by shocks; therefore the velocity spectra are steeper than the Kolmogorov slope of $-5 / 3$ and closely resemble the Burgers -2 scaling (Kritsuk et al. 2006b). The physics of threedimensional supersonic turbulence is, however, quite different from burgulence (Frisch \& Bec 2001) as the solenoidal velocity component always remains dominant (Pouquet et al. 1991; Pavlovski et al. 2006; Kritsuk et al. 2007; Pan et al. 2009; Schmidt et al. 2009; Kritsuk et al. 2010). At sonic Mach numbers $M_{\mathrm{S}}>3$, strong shock interactions and associated nonlinear instabilities create sophisticated multi-scale pattern of nested $\mathrm{U}$-shaped structures in dynamically active regions morphologically similar to what is observed in molecular clouds (Kritsuk et al. 2006a). Scaling of the first-order velocity structure functions $S_{1}(\delta \mathbf{u}) \sim \ell^{0.54}\left(\right.$ where $\delta \mathbf{u}(\ell)=\mathbf{u}(\mathbf{x})-\mathbf{u}(\mathbf{x}+\hat{\mathbf{e}} \ell), S_{p}(\delta \mathbf{u})=\left\langle[\delta \mathbf{u}(\ell)]^{p}\right\rangle$ and $\langle\ldots\rangle$ indicates averaging over an ensemble of random point pairs separated by the lag $\ell$ ) obtained in simulations (Kritsuk et al. 2007) is similar to the velocity scaling observed in molecular clouds $S_{1}(\delta \mathbf{u}) \sim \ell^{0.56}$ (Heyer \& Brunt 2004). Simulations also support the concept of (lossy) energy cascade in compressible turbulence (e.g., Vázquez-Semadeni et al. 2003), suggesting that the kinetic energy directly lost in shocks constitutes a small fraction of the total energy dissipation. The fact that the Richardson-Kolmogorov cascade picture does approximately hold for supersonic turbulence follows from the linear scaling of the third-order structure function of the mass-weighted velocity, $S_{3}(\delta \sqrt[3]{\rho} \mathbf{u}) \sim \ell$, indicating constant turbulent energy transfer rate across the hierarchy of scales (Kritsuk et al. 2007; Kowal \& Lazarian 2007; Schwarz et al. 2010). The power spectra of $\sqrt[3]{\rho} \mathbf{u}$, accordingly, demonstrate the Kolmogorov scaling independent of the Mach number (Kritsuk et al. 2007; Schmidt et al. 2008; Kritsuk et al. 2009; Federrath et al. 2010; Price \& Federrath 2010). It seems that this result can be also extended to supersonic MHD turbulence, where the incompressible 4/3-law of Politano \& Pouquet (1998) also approximately holds in its scaling part, $S_{\|, 3}^{ \pm} \equiv\left\langle\delta \mathbf{Z}_{\|}^{\mp}(\ell)\left[\delta \mathbf{Z}_{i}^{ \pm}(\ell)\right]^{2}\right\rangle \sim \ell$ (here $\delta \mathbf{Z}_{\|}(\ell) \equiv[\mathbf{Z}(\mathbf{x}+\hat{\mathbf{e}} \ell)-\mathbf{Z}(\mathbf{x})] \cdot \hat{\mathbf{e}}$, and $\hat{\mathbf{e}} \ell$ is the displacement vector), if reformulated in terms of the mass-weighted Elsässer fields $\mathbf{Z}^{ \pm} \equiv \rho^{1 / 3}(\mathbf{u} \pm \mathbf{B} / \sqrt{4 \pi \rho})$ (Kritsuk et al. 2009). The presence of magnetic field effectively reduces compressibility of the gas making the velocity spectra more shallow with slopes approaching the Iroshnikov-Kraichnan index of -1.5 in trans-Alfvénic flows. The observed spectral slope of about -1.8 for the Perseus molecular cloud is thus consistent with the super-Alfvénic turbulence regime dominant in that cloud (Padoan et al. 2006).

Recent results from numerical experiments on highly compressible turbulence stimulated theorists to reconsider the steady-state statistics of turbulence in the inertial interval. Falkovich et al. (2010) have shown that the Kolmogorov 4/5-law is a particular case of the general relation on the current-density correlation function. They derived an analog of the flux relation for compressible turbulence that can be used as a test for direct numerical simulations and as a guide for the development of subgrid scale models for astrophysical turbulence (Schmidt \& Federrath 2010). 
Most of the recent sub-mesoscale models belong to a class of so-called converging (or colliding) flows of diffuse Hi originally developed to study thermal, dynamic, and gravitational instabilities in shock-bounded slabs (Hunter et al. 1986; Vishniac 1994; Walder \& Folini 1998; Folini et al. 2010). These models remain popular as a framework to directly simulate star formation in molecular clouds (Vázquez-Semadeni et al. 2006; Hennebelle \& Inutsuka 2006; Vázquez-Semadeni et al. 2007; Hennebelle et al. 2008; Inoue \& Inutsuka 2008-09; Heitsch et al. 2008-09; Banerjee et al. 2009; Niklaus et al. 2009; Audit \& Hennebelle 2010; Rosas-Guevara et al. 2010). Recent numerical experiments with converging flows have demonstrated strong sensitivity of results to adopted initial and boundary conditions as well as to model parameters that control the density of colliding gas streams, mean thermal pressure, orientation and strength of the mean magnetic field, levels and character of "turbulence" at infinity, etc. All these parameters live their unique imprints in the statistics of derived stellar populations and any comprehensive parameter study based on computational modeling in this framework would be prohibitively expensive.

One way to circumvent these difficulties is to exploit Prigogine's concept of selforganization in non-equilibrium nonlinear dissipative systems (Nicolis \& Prigogine 1977) in application to the ISM (e.g., Biglari \& Diamond 1989). With this approach, one can use interstellar turbulence as an agent that imposes "order" in the form of coherent structures and correlations between various flow fields emerging in a simple periodic box simulation when a statistical steady state develops. In this case, the initial conditions are no longer important, instead the steady state would provide the "correct" turbulent initial conditions for star formation when self-gravity is turned on. While this idea is not new,$\dagger$ it remained largely undeveloped so far. In the following sections we will discuss this concept in more detail and report first results from a series of MHD simulations of turbulent multiphase ISM with the piecewise parabolic method on a local stencil (PPML; Ustyugov et al. 2009).

\section{Self-organization in the magnetized multiphase ISM}

In out numerical experiments, we treat the ISM as a turbulent, driven system, with kinetic energy being injected at the largest scales by supernova explosions, shear associated with differential rotation of the galactic disk, gas accretion onto the disk, etc. (Mac Low \& Klessen 2004; Klessen \& Hennebelle 2010). This kinetic energy is then being transferred from large to small scales in a cascade-like fashion. As our models include a mean magnetic field, $B_{0}$, some part of this kinetic energy gets stored in the turbulent magnetic field component, $b$, generated by stretching, twisting, and folding of magnetic field lines. The ISM is also exposed to the far-ultraviolet (FUV) background radiation due to OB associations of quickly evolving massive stars that form in molecular clouds. This FUV radiation is the main source of energy input for the neutral gas phases and this volumetric thermal energy source is in turn balanced by radiative cooling (Wolfire et al. 2003). The ISM is thus exposed to various energy fluxes, and self-organization arises as a result of the relaxation through nonlinear interactions of different physical constituents of the system subject to usual MHD constraints in the form of conservation laws. In this picture, molecular clouds with their hierarchical internal structure form as dissipative structures that represent active regions of highly intermittent turbulent cascade that drain the kinetic energy supplied by the driving forces.

$\dagger$ See, for instance, summary of the panel discussion on Phases of the ISM during the 1986 Grand Teton Symposium in Wyoming (Shull 1987). 
Table 1. Model parameters.

\begin{tabular}{lccccccc}
\hline Model & $N^{3}$ & $\begin{array}{c}n_{0} \\
\mathrm{~cm}^{-3}\end{array}$ & $\begin{array}{c}u_{\mathrm{rms}, 0} \mathrm{~km} / \mathrm{s} \\
\mathrm{kg}\end{array}$ & $\begin{array}{c}B_{0} \\
\mu \mathrm{G}\end{array}$ & $\beta_{\mathrm{th}, 0}$ & $\beta_{\mathrm{turb}, 0}$ & $M_{A, 0}$ \\
\hline $\mathrm{A}$ & $512^{3}$ & 5 & 16 & 9.54 & 0.2 & 3.3 & 1.3 \\
$\mathrm{~B}$ & $512^{3}$ & 5 & 16 & 3.02 & 2 & 33 & 4.0 \\
$\mathrm{C}$ & $512^{3}$ & 5 & 16 & 0.95 & 20 & 330 & 13 \\
$\mathrm{D}$ & $256^{3}$ & 2 & 16 & 3.02 & 2 & 13.2 & 2.6 \\
$\mathrm{E}$ & $256^{3}$ & 5 & 7 & 3.02 & 2 & 8.3 & 2.0 \\
\hline
\end{tabular}

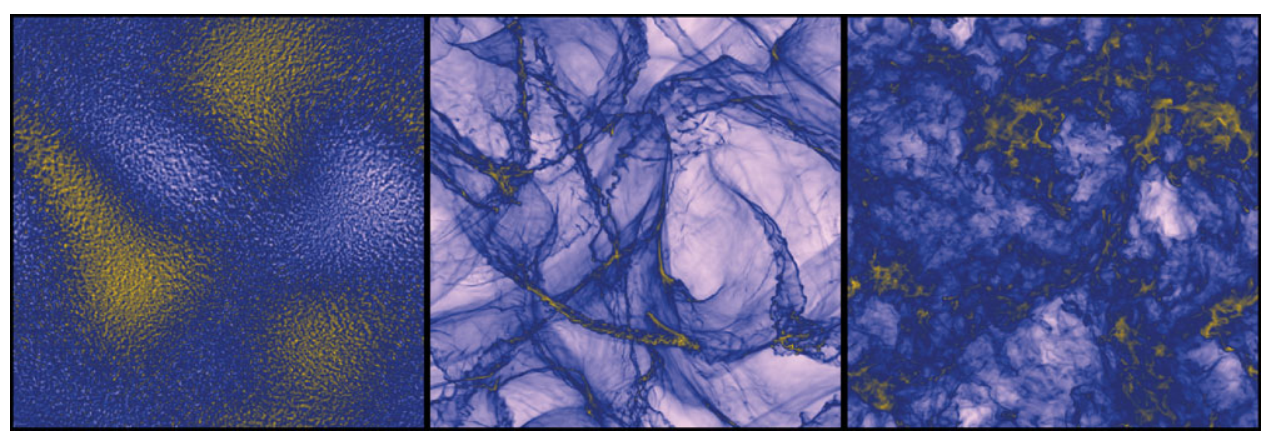

Figure 1. Three snapshots of projected gas density in model A taken at $t=2,3$, and 4 Myr. The white-blue-yellow colors correspond to low-intermediate-high projected density values.

\section{Modeling the formation of molecular clouds}

To illustrate these ideas, we consider a set of simple periodic box models, which ignore gas stratification and differential rotation in the disk and employ an artificial large-scale solenoidal force to mimic the supply of kinetic energy from various galactic sources. This naturally leads to an upper bound on the box size, $L$, which determines our choice of $L=200$ pc. Our models are, thus, fully defined by the following three parameters: the mean gas density in the box, $n_{0}$; the rms velocity, $u_{\mathrm{rms}, 0}$; and the mean magnetic field strength, $B_{0}$. All three would ultimately depend on $L$; Table 1 provides the summary of parameters for models A, B, C, D, and E assuming $L=200 \mathrm{pc}$. The table also gives the grid resolution, $N$, the initial values for plasma beta, $\beta_{\mathrm{th}, 0} \equiv 8 \pi p_{0} / B_{0}^{2}$, turbulent beta, $\beta_{\text {turb }, 0} \equiv 8 \pi \rho_{0} u_{\mathrm{rms}, 0}^{2} / B_{0}^{2}$, and Alfvénic Mach number, $M_{A, 0}=\left(4 \pi \rho_{0}\right)^{1 / 2} u_{\mathrm{rms}, 0} / B_{0}$, where $p_{0}$ is the initial thermal pressure of the gas (see the phase diagram in Fig. 2 for more detail).

We initiate our numerical experiments with a uniform gas distribution in the computational domain. An addition of small random isobaric density perturbations at $t=0$ triggers a phase transition in the thermally bi-stable gas that quickly turns $\sim 25-65 \%$ of the gas mass into the stable cold phase (CNM with temperature below $T=184 \mathrm{~K}$ ), while the rest of the mass is shared between the unstable and stable warm gas (WNM). In models A, B, and C, CNM and WNM each contain roughly $\sim 50 \%$ of the total HI mass in agreement with observations (Heiles \& Crutcher 2005). We then turn on the forcing and after a few large-eddy turn-over times the simulation approaches a statistical steady state. If we replace this two-stage initiation process with a one-stage procedure by turning the driving on at $t=0$, the properties of the steady state remain unchanged. Figure 1 illustrates this evolutionary sequence for the two-stage case with three snapshots of projected gas density for model A. The left panel shows two-phase medium at $t=2$ Myr right before we turn on the forcing; the panel in the middle illustrates an early stage of turbulization with transient "colliding flows" at $t=3 \mathrm{Myr}$. The right panel 

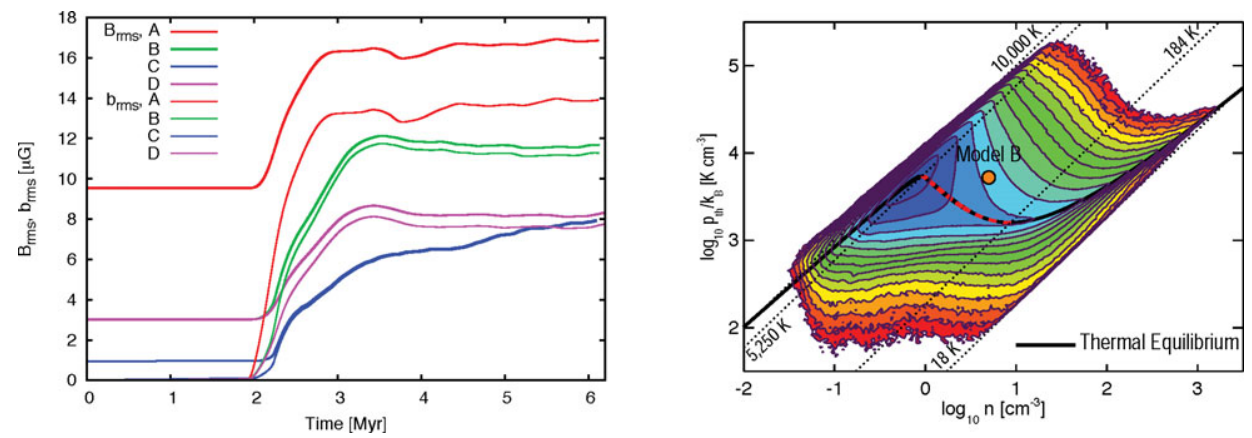

Figure 2. Time evolution of the rms magnetic field strength, $B_{\mathrm{rms}}$, and its turbulent component, $b_{\mathrm{rms}}$, for models A, B, C, and D (left panel). Phase diagram (thermal pressure versus gas density) for model $\mathrm{B}$ at $t=5 \mathrm{Myr}$ (right panel).
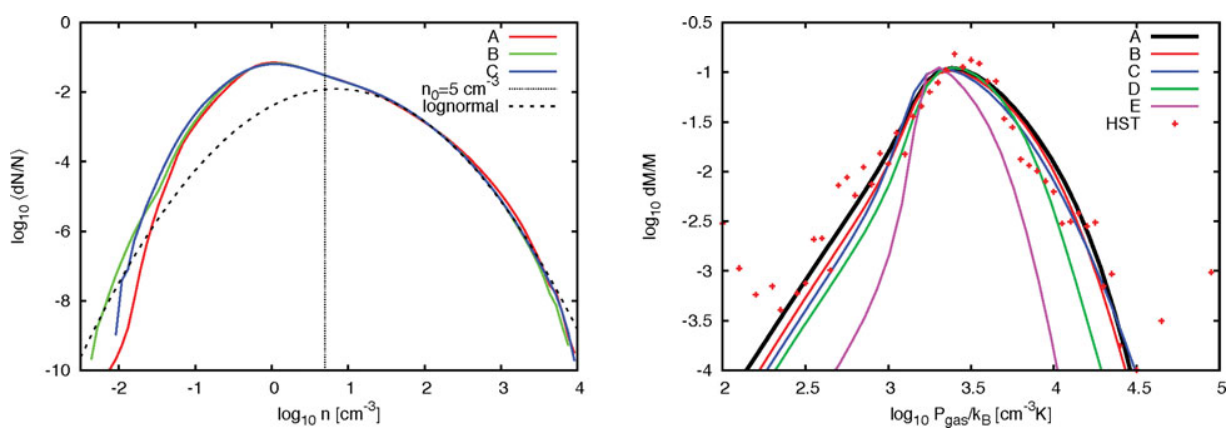

Figure 3. Time-average density distributions for fully developed turbulence in models A, B, and $\mathrm{C}$ (left panel) and time-average mass-weighted thermal pressure distributions for models $\mathrm{A}$, $\mathrm{B}, \mathrm{C}$, and D (right panel); see text for more detail.

shows the projected density at $t=4 \mathrm{Myr}$ for a statistically developed turbulent state. Molecular clouds can be seen in the right panel as filamentary brown-to-yellow structures (note that these are morphologically quite different from the transient dense structures in the middle panel). The rms magnetic field is amplified by the forcing and saturates when the relaxation in the system results in a steady state, see Fig. 2. The level of saturation depends on $B_{0}$ and on the rate of kinetic energy injection by the large-scale force, which is in turn determined by $u_{\mathrm{rms}}$ and $n_{0}$. This level can be easily controlled with the model parameters. In the saturated regime, models A and B tend to establish energy equipartition $\left(E_{\mathrm{K}} \sim E_{\mathrm{M}}\right)$, while the saturation level of magnetic energy in model $\mathrm{C}$ is a factor of $\sim 3$ lower than the equipartition level. The mean thermal energy also gets a slight boost due to forcing, but remains subdominant in all the models. A typical phase diagram is shown in Fig. 2. The contours indicate constant levels of volume fraction for different regimes of the thermal pressure, $p_{\text {th }}$, and density, $n$, separated by factors of 2 . About $23 \%$ of the domain volume is filled with the stable warm phase at $T>5250 \mathrm{~K}$, the stable cold phase $(T<184 \mathrm{~K})$ occupies $\sim 7 \%$, and $\sim 70 \%$ of the volume resides in the thermally unstable regime at intermediate temperatures. The big orange dot at the center indicates the (forgotten) initial conditions for models $\mathrm{A}, \mathrm{B}, \mathrm{C}$, and $\mathrm{E}$. The phase diagram indicates that turbulence supports an enormously wide range of thermal pressures and also that $p_{\text {th }}$ in the molecular gas $\left(n>100 \mathrm{~cm}^{-3}\right)$ is higher than that in the diffuse ISM, even though self-gravity is ignored in the model. 

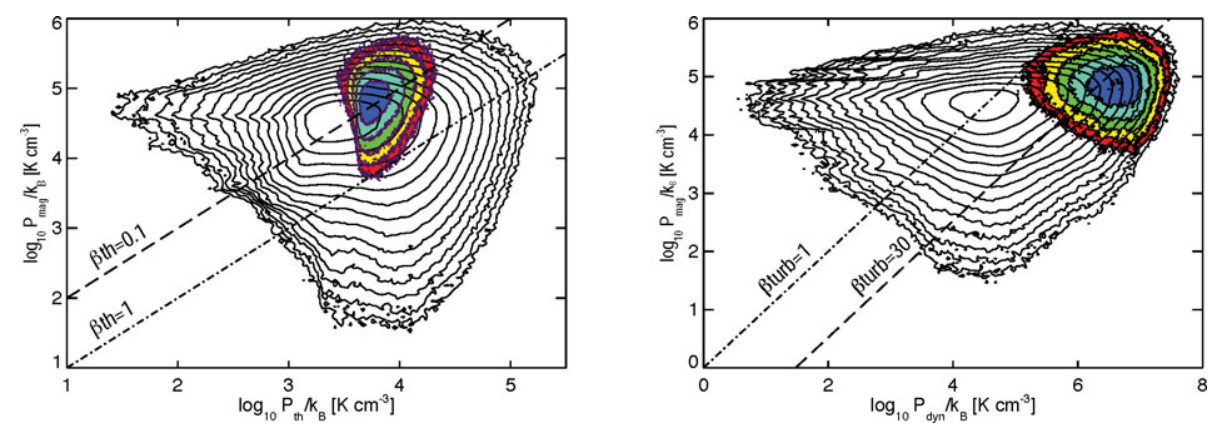

Figure 4. Distributions of magnetic pressure vs. thermal (left) and dynamic (right) pressure for a snapshot from model $\mathrm{B}$ at $t=5 \mathrm{Myr}$.
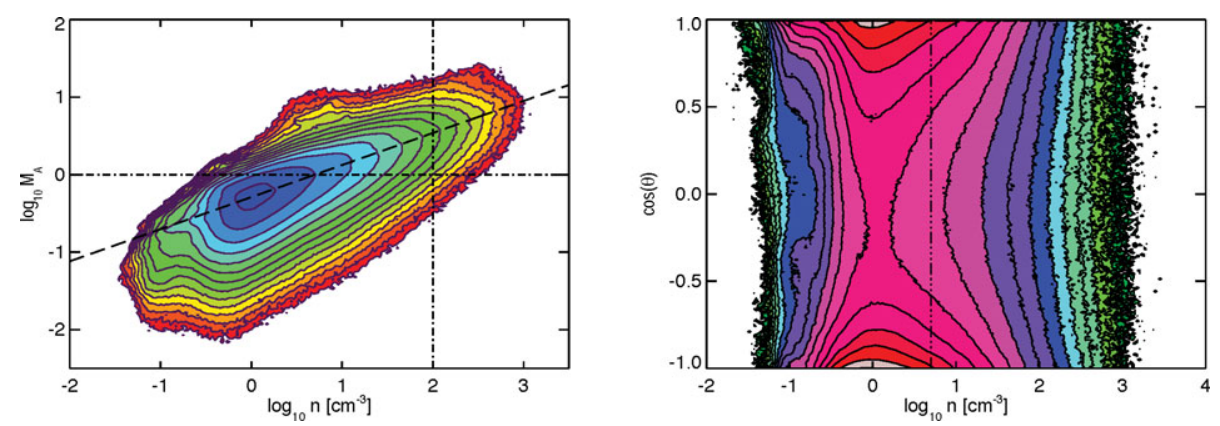

Figure 5. Distributions of the Alfvénic Mach number and the cosine of the alignment angle vs. density for a snapshot from model A at $t=5 \mathrm{Myr}$.

Figure 3 (left) shows the time-average density PDFs for models A, B, and C in the steady state. The effect of magnetic field on the density PDF is apparently very weak on average, and the high-density part of the PDF can be well approximated by a lognormal as in the isothermal case. The signature of the two stable thermal phases in the PDF is smeared by the (relatively) high level of turbulence, $u_{\mathrm{rms}}(100 \mathrm{pc})=16 \mathrm{~km} / \mathrm{s}$ (Brunt \& Heyer 2004), but the overall shape of the distribution is not lognormal. The distribution of thermal pressure for models $\mathrm{A}, \mathrm{B}$, and $\mathrm{C}$ spans about 6 dex leaving no room for the old pressure-supported cloud picture in the violent ISM. All distributions match the characteristic pressure typical for the Milky Way disk at the solar radius and show only weak dependence on $B_{0}$, while the width of the distribution remains sensitive to $u_{\mathrm{rms}}$ and $n_{0}$. Figure 3 (right) shows how the mass-weighted pressure distributions obtained in our models compare with the distribution reconstructed from high-resolution UV spectra of hot stars in the HST archive (Jenkins \& Tripp 2010). It seems that models with $u_{\mathrm{rms}}=16 \mathrm{~km} / \mathrm{s}$ reproduce both the shape and the width of the observed distribution quite nicely, while a lower turbulence level in model E makes that model distribution too narrow.

These numerical experiments allow us to probe the levels of magnetic field strength in molecular clouds that form self-consistently in the magnetized turbulent diffuse ISM. In the left panel of Fig. 4, we show a scatter plot of magnetic vs. thermal pressure for model B at $t=5 \mathrm{Myr}$. The black contour lines show the distribution for the whole domain, which is centered at $\beta_{\mathrm{th}} \approx 0.1$. The subset of cells representing the molecular gas $(T<100 \mathrm{~K}$ and $n>100 \mathrm{~cm}^{-3}$, color contour plot) shows very similar mean values of $\beta_{\mathrm{th}}$. This indicates a 
target plasma beta for realistic isothermal molecular cloud turbulence simulations. The right panel of the same Figure shows a scatter plot of magnetic vs. dynamic pressure for the same snapshot from the same model. The distribution for the whole domain is centered at $\beta_{\text {turb }} \approx 1$ because kinetic and magnetic energy levels are close to equipartition on average. At the same time, the subset of cells representing the molecular gas (color contours) shows a distribution centered at $\beta_{\text {turb }} \approx 30$ meaning that turbulence in the molecular gas is super-Alfvénic. Figure 5, left panel, shows the distribution of Alfvénic Mach number, $M_{\mathrm{A}}$, as a function of density for the strongly magnetized model $\mathrm{A}$ that further supports this result. There is a clear positive correlation, $M_{\mathrm{A}} \sim n^{0.4}$, indicated by the dashed line and most of the dense material $\left(n>100 \mathrm{~cm}^{-3}\right)$ clearly falls into the super-Alfvénic part of the distribution.

A key to understanding the origin of this super-Alfvénic regime in the cold and dense molecular gas lies in the process of self-organization in magnetized ISM turbulence that we briefly introduced in Section 2. The statistical steady state that our models attain on a time-scale of a few million years is characterized by a certain degree of alignment between the velocity and magnetic field lines. Figure 5, right panel, shows the distribution of the cosine of the alignment angle, $\cos \theta \equiv \mathbf{B} \cdot \mathbf{u} /(B u)$, for model A at $t=5 \mathrm{Myr}$. The contours indicate a saddle-like structure of this probability distribution with a strong alignment regime $(\cos \theta= \pm 1)$ in the WNM at and around $n \sim 1 \mathrm{~cm}^{-3}$. This means that compressions in the WNM gas, which is on average trans-Alfvénic (e.g., $M_{\mathrm{A}} \in[0.6,0.9]$ in model A), occur preferentially along the field lines. If molecular clouds form in the turbulent ISM via large-scale compression of the diffuse $\mathrm{HI}$, then turbulence in such molecular clouds can only be super-Alfvénic, see also Padoan et al. (2010).

\section{Conclusion}

Rapid development of computational astrophysics in the recent years enabled progress in understanding the basics of interstellar turbulence. These new advances will help us to move forward with direct star formation simulations from turbulent initial conditions.

Acknowledgements. This research was supported in part by the National Science Foundation through grants AST-0607675, AST-0808184, and AST-0908740, as well as through TeraGrid resources provided by NICS and SDSC (MCA07S014) and through DOE Office of Science INCITE-2009 and DD-2010 awards allocated at NCCS (ast015/ast021).

\section{References}

Audit, E. \& Hennebelle, P. 2010, A\& A, 511, A76

de Avillez, M. A. \& Breitschwerdt, D. 2007, ApJL, 665, L35

de Avillez, M. A. \& Breitschwerdt, D. 2005, A\& A, 436, 585

Banerjee, R., Vázquez-Semadeni, E., Hennebelle, P., \& Klessen, R. S. 2009, MNRAS, 398, 1082

Biglari, H. \& Diamond, P. H. 1989, Physica D Nonlinear Phenomena, 37, 206

Elmegreen, B. G. \& Scalo, J. 2004, ARA\&A, 42, 211

Falkovich, G., Fouxon, I., \& Oz, Y. 2010, Journal of Fluid Mechanics, 644, 465

Federrath, C., Roman-Duval, J., Klessen, R. S., Schmidt, W., \& Mac Low, M.-M. 2010, A\&্A, $512, \mathrm{~A} 81$

Folini, D., Walder, R., \& Favre, J. M. 2010, ASP Conf. Ser., 429, 9

Frisch, U. \& Bec, J. 2001, New Trends in Turbulence, 341

Gazol, A. \& Kim, J. 2010, ApJ, 723, 482

Gazol, A., Luis, L., \& Kim, J. 2009, ApJ, 693, 656

Heiles, C. \& Crutcher, R. 2005, Cosmic Magnetic Fields, Lect. Notes Phys., 664, 137 
Heitsch, F., Hartmann, L., Slyz, A., Devriendt, J., \& Burkert, A. 2008, ApJ, 674, 316

Heitsch, F., Stone, J. M., \& Hartmann, L. W. 2009, ApJ, 695, 248

Hennebelle, P., Banerjee, R., Vázquez-Semadeni, E., Klessen, R. S., \& Audit, E. 2008, A\&A, 486, L43

Hennebelle, P. \& Inutsuka, S.-i. 2006, ApJ, 647, 404

Heyer, M. H. \& Brunt, C. M. 2004, ApJL, 615, L45

Hunter, J. H., Jr., Sandford, M. T., II, Whitaker, R. W., \& Klein, R. I. 1986, ApJ, 305, 309

Inoue, T. \& Inutsuka, S.-i. 2009, ApJ, 704, 161

Inoue, T. \& Inutsuka, S.-i. 2008, ApJ, 687, 303

Jenkins, E. B. \& Tripp, T. M. 2010, to appear in ApJ

Joung, M. K. R. \& Mac Low, M.-M. 2006, ApJ, 653, 1266

Joung, M. R., Mac Low, M.-M., \& Bryan, G. L. 2009, ApJ, 704, 137

Kissmann, R., Kleimann, J., Fichtner, H., \& Grauer, R. 2008, MNRAS, 391, 1577

Klessen, R. S. \& Hennebelle, P. 2010, A\& A, 520, A17

Korpi, M., Brandenburg, A., Shukurov, A., Tuominen, I., \& Nordlund, Å. 1999, ApJL, 514, L99

Kowal, G. \& Lazarian, A. 2007, ApJL, 666, L69

Kritsuk, A. G., Ustyugov, S. D., Norman, M. L., \& Padoan, P. 2010, ASP Conf. Ser., 429, 15

Kritsuk, A. G., Ustyugov, S. D., Norman, M. L., \& Padoan, P. 2009, ASP Conf. Ser., 406, 15

Kritsuk, A. G., Norman, M. L., Padoan, P., \& Wagner, R. 2007, ApJ, 665, 416

Kritsuk, A. G., Norman, M. L., \& Padoan, P. 2006a, ApJL, 638, L25

Kritsuk, A. G., Wagner, R., Norman, M. L., \& Padoan, P. 2006b, ASP Conf. Ser., 359, 84

Mac Low, M.-M., Balsara, D. S., Kim, J., \& de Avillez, M. A. 2005, ApJ, 626, 864

Mac Low, M.-M. \& Klessen, R. S. 2004, Rev. Mod. Phys., 76, 125

Malhotra, S. 1995, ApJ, 448, 138

Nicolis, G. \& Prigogine, I., Self-Organization in Nonequilibrium Systems, Wiley, New York, 1977

Niklaus, M., Schmidt, W., \& Niemeyer, J. C. 2009, A\&A, 506, 1065

Padoan, P., et al. 2010, AIP Conf. Proc., 1242, 219

Padoan, P., Juvela, M., Kritsuk, A., \& Norman, M. L. 2006, ApJL, 653, L125

Pan, L., Padoan, P., \& Kritsuk, A. G. 2009, Phys. Rev. Lett., 102, 034501

Pavlovski, G., Smith, M. D., \& Mac Low, M.-M. 2006, MNRAS, 368, 943

Politano, H. \& Pouquet, A. 1998, Geophys. Res. Lett., 25, 273

Pouquet, A., Passot, T., \& Leorat, J. 1991, Proc. IAU Symp. 147: Fragmentation of Molecular Clouds and Star Formation, p. 101

Price, D. J. \& Federrath, C. 2010, MNRAS, 406, 1659

Rosas-Guevara, Y., Vázquez-Semadeni, E., Gómez, G., \& Jappsen, A. 2010, MNRAS, 406, 1875

Schmidt, W. \& Federrath, C. 2010, arXiv:1010.4492

Schmidt, W., Federrath, C., Hupp, M., Kern, S., \& Niemeyer, J. C. 2009, A $\& A$ A, 494, 127

Schmidt, W., Federrath, C., \& Klessen, R. 2008, Phys. Rev. Lett., 101, 194505

Schwarz, C., Beetz, C., Dreher, J., \& Grauer, R. 2010, Physics Letters A, 374, 1039

Seifried, D., Schmidt, W., \& Niemeyer, J. C. 2010, arXiv:1009.2871

Shull, J. M. 1987, Interstellar Processes, 134, 225

Solomon, P. M., Rivolo, A. R., Barrett, J., \& Yahil, A. 1987, ApJ, 319, 730

Tasker, E. J. \& Bryan, G. L. 2006, ApJ, 641, 878

Tasker, E. J. \& Bryan, G. L. 2008, ApJ, 673, 810

Ustyugov, S., Popov, M., Kritsuk, A., \& Norman, M. 2009, J. Comp. Phys., 228, 7614

Vázquez-Semadeni, E., et al. 2007, ApJ, 657, 870

Vázquez-Semadeni, E., Ryu, D., Passot, T., González, R. F., \& Gazol, A. 2006, ApJ, 643, 245

Vázquez-Semadeni, E., Ballesteros-Paredes, J., \& Klessen, R. 2003, ASP Conf. Ser., 287, 81

Vázquez-Semadeni, E. \& Passot, T. 1999, Interstellar Turbulence, Proc. 2nd Guillermo Haro Conference, Eds. J. Franco and A. Carraminana. Cambridge University Press, p. 223

Vishniac, E. T. 1994, ApJ, 428, 186

Wada, K. 2008, ApJ, 675, 188

Walder, R. \& Folini, D. 1998, A\& A, 330, L21

Wolfire, M. G., McKee, C. F., Hollenbach, D., \& Tielens, A. G. G. M. 2003, ApJ, 587, 278 